\title{
Design and Modeling of RF Power Amplifiers with Radial Basis Function Artificial Neural Networks
}

\author{
Ali Reza Zirak \\ Laser \& Optics Research School, \\ NSTRI, \\ Tehran, Iran
}

\author{
Sobhan Roshani* \\ Young Researchers and Elite Club, Kermanshah Branch, \\ Islamic Azad University, \\ Kermanshah, Iran
}

\begin{abstract}
A radial basis function (RBF) artificial neural network model for a designed high efficiency radio frequency class-F power amplifier (PA) is presented in this paper. The presented amplifier is designed at $1.8 \mathrm{GHz}$ operating frequency with $12 \mathrm{~dB}$ of gain and $36 \mathrm{dBm}$ of $1 \mathrm{~dB}$ output compression point. The obtained power added efficiency (PAE) for the presented PA is $76 \%$ under $26 \mathrm{dBm}$ input power. The proposed RBF model uses input and DC power of the PA as inputs variables and considers output power as the output variable. The presented RBF network models the designed class-F PA as a block, which could be applied in circuit design. The presented model could be used to model any RF power amplifier. The obtained results show a good agreement between real data and predicted values by RBF model. The results clearly show that the presented RBF network is more precise than multilayer perceptron (MLP) model. According to the results, better than $84 \%$ and $92 \%$ improvement is achieved in MAE and RMSE, respectively.
\end{abstract}

Keywords-Amplifier model; artificial neural network (ANN); class-F amplifier; radial basis function; RF amplifier

\section{INTRODUCTION}

PAs are important elements, in any communication system, which consume a lot of power. Therefore, the efficiency is a very important factor in the design process. Considerable energy can be saved, by improving the efficiency of PAs [1-2]. The switching mode amplifiers are introduced to achieve high efficiency. Class-F PAs have become very popular in switching mode amplifiers, because of their high efficiency and output capability [3-5]. In the class-F PAs, Ideal drain efficiency could be obtained, due to the optimum drain voltage and drain current waveforms shape. Several methods have been introduced to model power amplifiers, such as Wiener [6] and Volterra series [7]. However, these mentioned models need approximation for obtaining the specifications of the device. Neural network based models are faster and more precise, compared with the mentioned modeling methods [8]. Any continuous nonlinear function could be approximated using ANNs. Therefore, ANNs can be used to model nonlinear circuits [9].

Power amplifiers are recently modeled, using artificial neural networks algorithms [10-12]. In [10], adaptive neurofuzzy inference system (ANFIS) is presented to model a PA. In this model, memory effect of the amplifier is not considered; however, the presented structure of the ANFIS is complicated. An ANN is used in [11] to model a $3 \mathrm{G}$ power amplifier. A large number of neurons are applied in this ANN, which makes the model complex. In this approach, the power spectral density (PSD) is obtained, however the other specifications of the power amplifier are not considered. Neural networks have also been used to model the other microwave components [1215]. In [12] a lowpass microstrip filter is modeled using multilayer perceptron (MLP) neural network with two hidden layers. The presented model in this research is used to predict the specifications of a lowpass microstrip filter; however, the applied model in this work is complex and uses a large number of neurons. A low voltage microwave LNA with operating frequency of $2.45 \mathrm{GHz}$ is modeled in [13]. In this approach MLP, RBF and ANFIS models are investigated and the small signal parameters are considered as the input of the networks. Channel dimensions of the transistors are considered as the output of the networks. However, the presented model can only be used in the applied circuit. In [16] a microstrip patch antenna is modeled and designed using RBF network. The results in this approach show that RBF network model is more accurate than the MLP network.

An efficient class-F amplifier is modeled by neural networks in [15]. The presented ANN model in this paper can predict the efficiency and output power of the amplifier. The MLP neural network training method for ANN is used in this research. The reported errors are acceptable, but it can be improved using other ANN methods.

Recently, the RBF networks have been used to model several microwave devices [17-18]. However they have not been applied to model power amplifiers in the way, which is used in this paper.

In this paper, at first a class-F switching amplifier is designed and simulated. Then, an RBF network is proposed to model the designed amplifier precisely. Finally, the presented model is compared with the conventional MLP ANN, which shows better performances. The presented model can be used as a sub-circuit model to describe the class-F power amplifier applications. For instance, it can be used in linearization method or Doherty power amplifier structures.

\section{DESIGN OF POWER AMPLIFIER}

Figure 1 shows the basic structure of the presented class-F PA, including a transistor, input/output matching network and DC supply voltage. The output matching network includes a parallel resonator circuit and a quarter wavelength transmission line [19]. The output network shapes the drain voltage and current waveforms to improve the efficiency [20]. Therefore, the output becomes short circuit in even harmonics, while it 
becomes open circuit in odd harmonics. The process of drain waveforms shaping improves the efficiency of class-F PA. There are one or more odd harmonics in voltage of the drain, such that it becomes approximately a square wave. In the contrary, even harmonics in the current of the drain make approximately a half sinusoidal waveform.

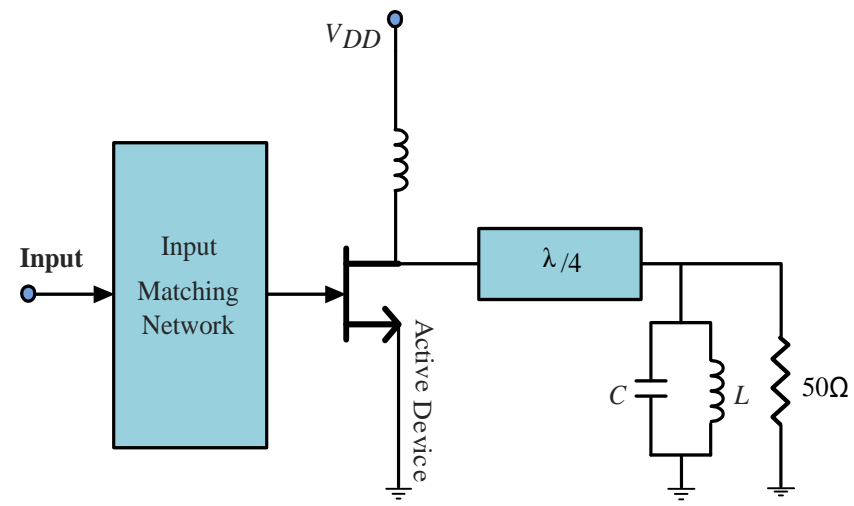

Fig. 1. Fundamental structure of the class-F PA

The designed matching circuit for the presented amplifier is shown in Fig. 2. According to this figure the applied microstrip transmission lines are shown as $\mathrm{T} 1$ - T10, which are used as harmonic control circuits. The power relations and the power added efficiency for the presented amplifier could be given as

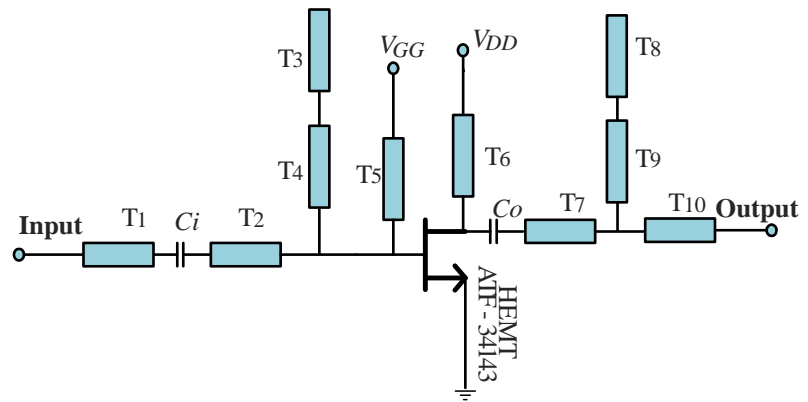

Fig. 2. Structure and matching networks of the designed amplifier

$$
\begin{gathered}
P A E=\frac{P_{\text {output }}-P_{\text {input }}}{P_{D C}} \\
P_{D C}=V_{D C} I_{D C} \\
P_{\text {output }}=\frac{V_{m}{ }^{2}}{2 R} .
\end{gathered}
$$

In above equations $P_{\text {output }}$ and $P_{D C}$ are output power and DC power, while $P_{\text {input }}$ is the input signal power. PAs convert the DC power and power of the input signal into output AC power. According to Fig. 2, an ATF 34143 HEMT is applied in the designed PA circuit. Simulation is carried out by advanced design system (ADS) software, which contains the device model. According to simulation results, $\mathrm{P} 1 \mathrm{~dB}$ of $36 \mathrm{dBm}$ is obtained. Also, the power gain and maximum PAE are $12 \mathrm{dBm}$ and $76 \%$ under the $26 \mathrm{dBm}$ input power. In the next section,
MLP and RBF networks will be introduced. Then, an RBF neural network model will be applied to the designed amplifier. The input and DC power of the power amplifier are assumed as input variables for the RBF network, while the output power is assumed as the output variable for the RBF network.

\section{MLP AND RBF MODELS}

ANN can be considered as a mathematical system consisting of simple processing elements called neurons. These neurons can be aligned in one or multiple layers. The MLP and RBF networks are the common architectures in neural networks. The MLP feed forward network has three layers, which the first and the second are the input layer and the hidden layer. The third layer is the output layer. There are several neurons in each layer of the network and the connections between them are weighted connections. The values of weights in each layer are obtained using training algorithm, which is commonly back propagation algorithm. Applying more neurons or more layers to the network may increase the precision of the model, but it cost complexity of the network and subsequently more time is needed to simulate the presented model.

The RBF network, introduced in 1988, is a feed forward ANN. The RBF network is an artificial neural network with radial basis activation functions, including three layers in the structure. The layers of the RBF network are similar to MLP network, but there is one hidden layer in the RBF network. Source nodes are in the input layer, and there are several neurons in the second layer (hidden layer), which are called the RBF centers. The outputs of the hidden neurons are summed, by the neurons in the output layer. Activation function of the units in the second layer is radial basis function. The structure of the typical RBF ANN is illustrated in Fig. 3, including two inputs and three layers. Input layer includes input nodes with the same number as the number of input vector [21]. The $i^{\text {th }}$ neuron output in the hidden layer could be given as

$$
q_{i}=\exp \left(-\frac{\left\|X_{i}-C_{i}\right\|^{2}}{\sigma_{i}{ }^{2}}\right),
$$

where $X$ is input vector, $C$ is basis function center and $\sigma_{i}$ is spread of Gaussian function. The estimated output can be written as0

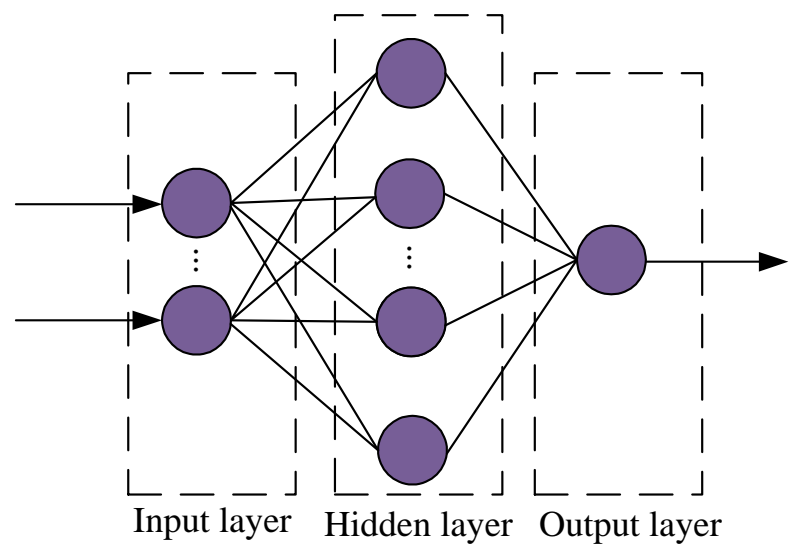

Fig. 3. Structure of the RBF ANN 


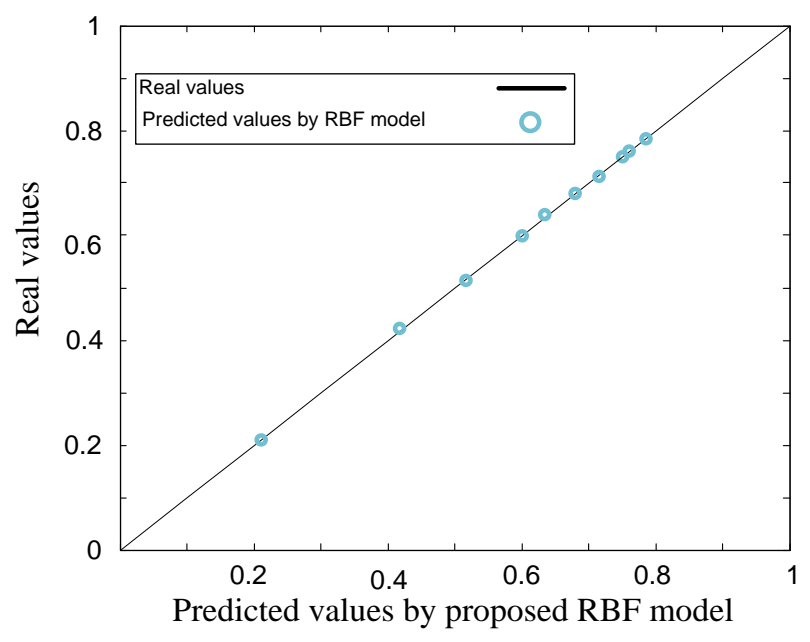

(a)

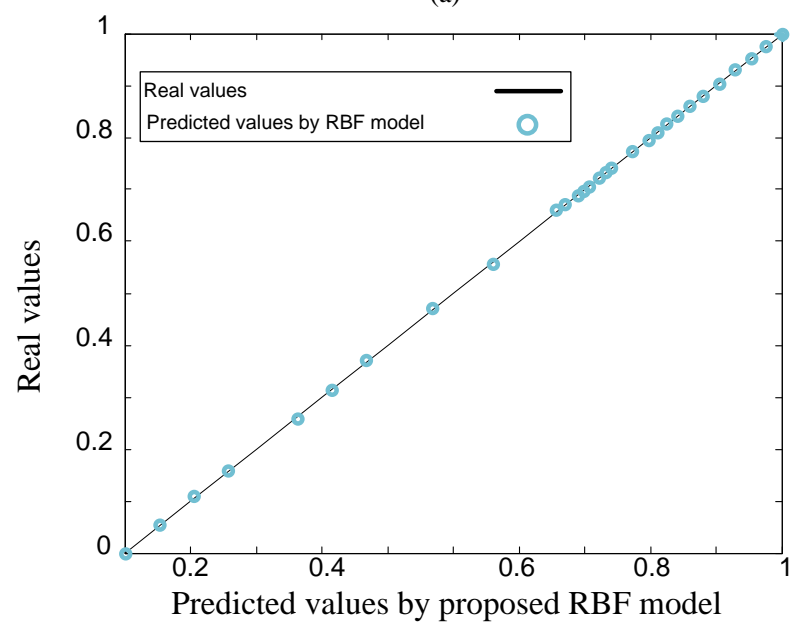

(b)

Fig. 4. Real and predicted output power results of (a) testing data and (b) training data, using RBF model

$$
y=\sum_{i=1}^{n} w_{i} q_{i}
$$

The connecting weights are shown with $w_{i}$ in equation (5). The input power and DC power are assumed as input variables of the network, while, the output power is assumed as the output variable of the network. About 75 percent of data set is used for training, while 25 percent is used for testing process of the proposed model in RBF network. Detailed results of the $\mathrm{RBF}$ network are given in Table 1 . The mean absolute error (MAE) and also the root mean square error (RMSE) for the presented network are given as

$$
\begin{gathered}
M A E \%=100 \times \frac{1}{N} \sum_{i=1}^{N}\left|Y_{R i}-Y_{P i}\right| \\
R M S E=\sqrt{\frac{\sum_{i=1}^{N}\left(Y_{R i}-Y_{P i}\right)^{2}}{N}}
\end{gathered}
$$

where $N$ is data number. The parameters $Y_{R i}$ and $Y_{P i}$ represent the real and predicted output of the presented ANN, respectively. A comparison between real and predicted output power results of training and testing data for the RBF network is illustrated in Fig. 4. Obtained errors of the presented RBF model, compared to the MLP model are summarized in Table 1. The MAE and the RMSE errors of the RBF network could be achieved using equations (6) and (7), respectively.

Real and predicted output power of the PA versus index number of each input for testing and training data using RBF network are illustrated in Fig. 5. According to Figs. 4 and 5, the predicted output power by RBF model is close to the real results. These results verify the accurate applicability of RBF network as a reliable model to predict the output power, using the input and DC power.

Real and predicted output power versus input power for training and testing data are depicted in Fig. 6. As shown in the figure, the presented amplifier is modeled precisely using the proposed RBF network. The real and predicted PAE versus input power for training and testing data are depicted in Fig. 7. Only data in the operating range of the presented amplifier are illustrated in Figs. 6 and 7. As shown, the main specifications of the designed amplifier are predicted using the presented model. Test and train data of the proposed RBF network are given in Table 2 and Table 3, respectively. Input power and DC power, which are the inputs of the network are shown in the first and second columns, while real output power and network predicted out power are compared in third and fourth columns. The predicted data have negligible deference with the real data, which verifies the presented model. 39 samples are selected for the network, which about $75 \%$ of the samples are considered for training and $25 \%$ are chosen for testing process of the proposed RBF network.

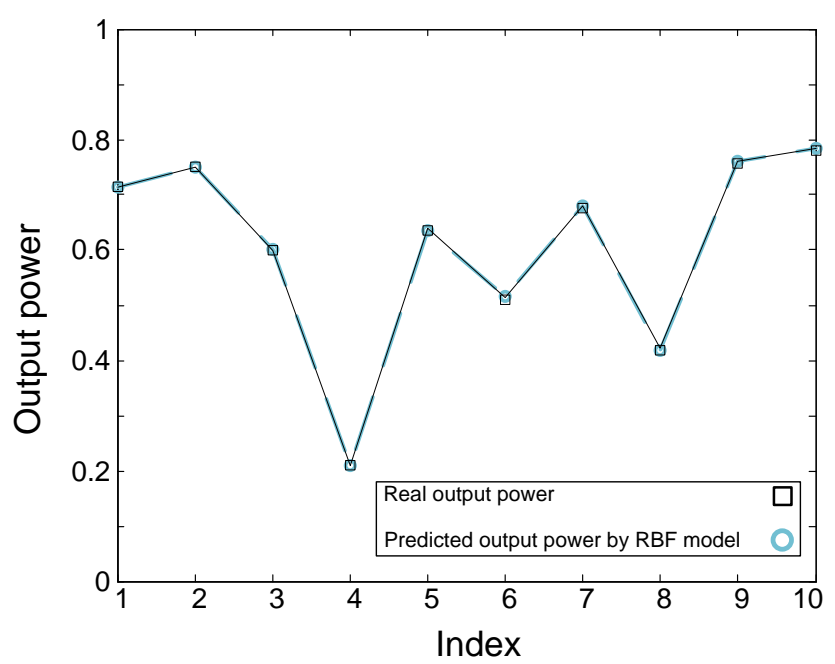

(a) 


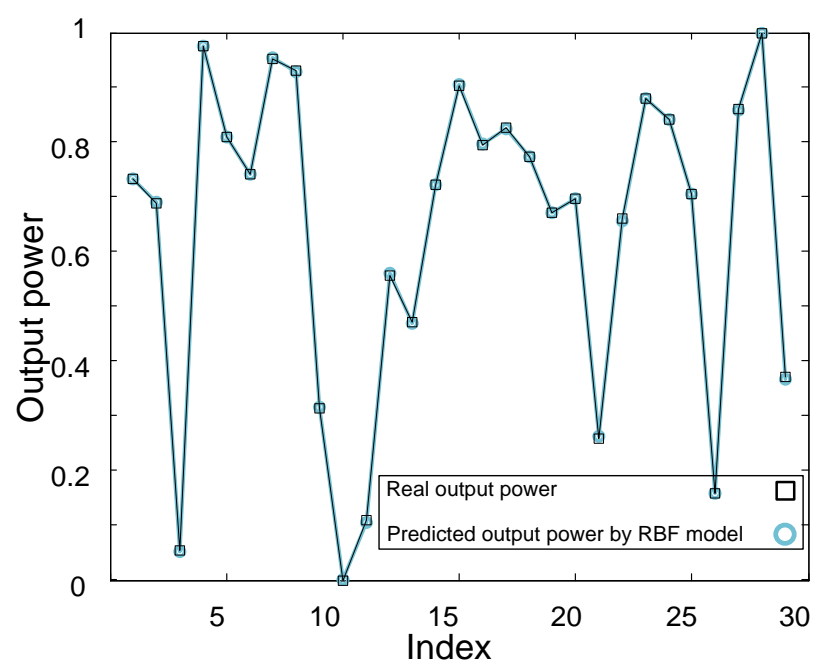

(b)

Fig. 5. Real and predicted output power of the PA versus index number of each input for (a) testing data and (b) training data, using RBF model

TABLE I. THE OBtAINED ERRORS OF THE PROPOSED RBF NETWORK

\begin{tabular}{crcc}
\hline Error & Train data & Test data & Test data \\
\hline & RBF & RBF & ANN[15] \\
\hline RMSE & 0.001 & 0.002 & 0.0275 \\
MAE\% & 0.131 & 0.311 & 1.9882 \\
\hline
\end{tabular}

TABLE II. TeSt Data of THE PROPOSEd RBF Network

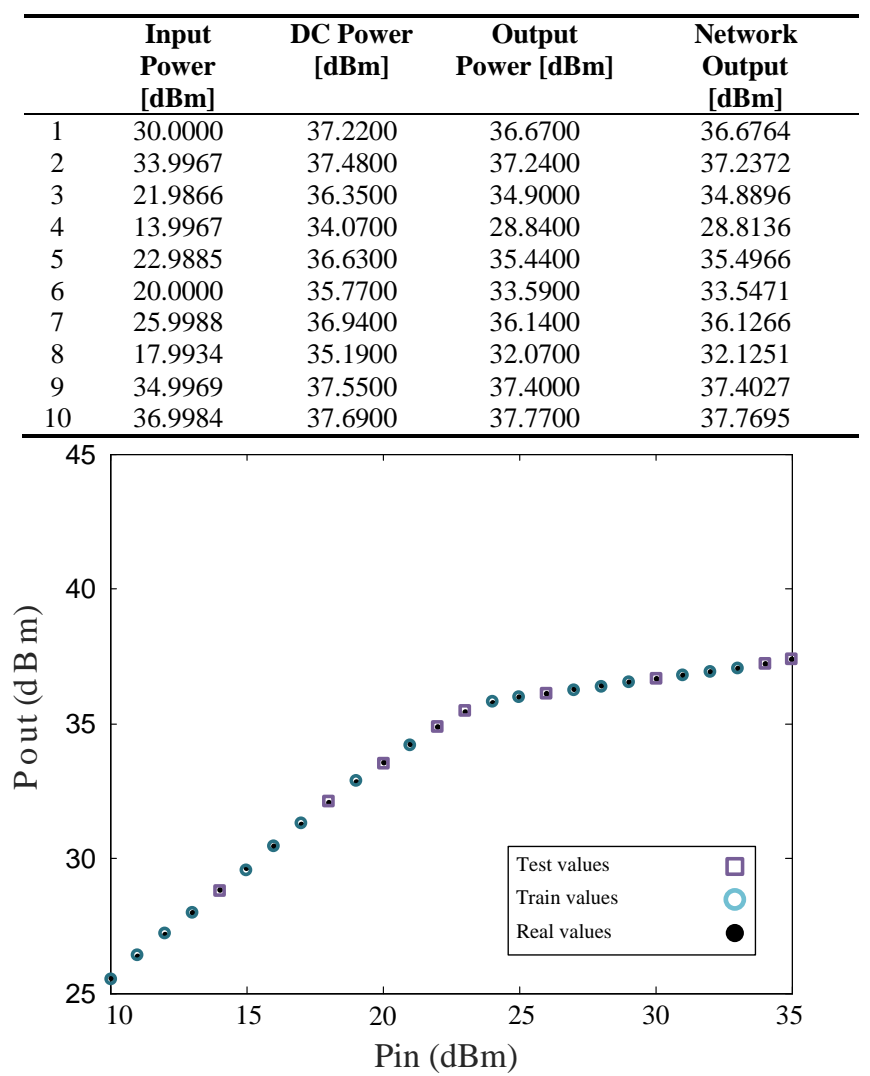

Fig. 6. Real and predicted output power versus input power for training and testing data

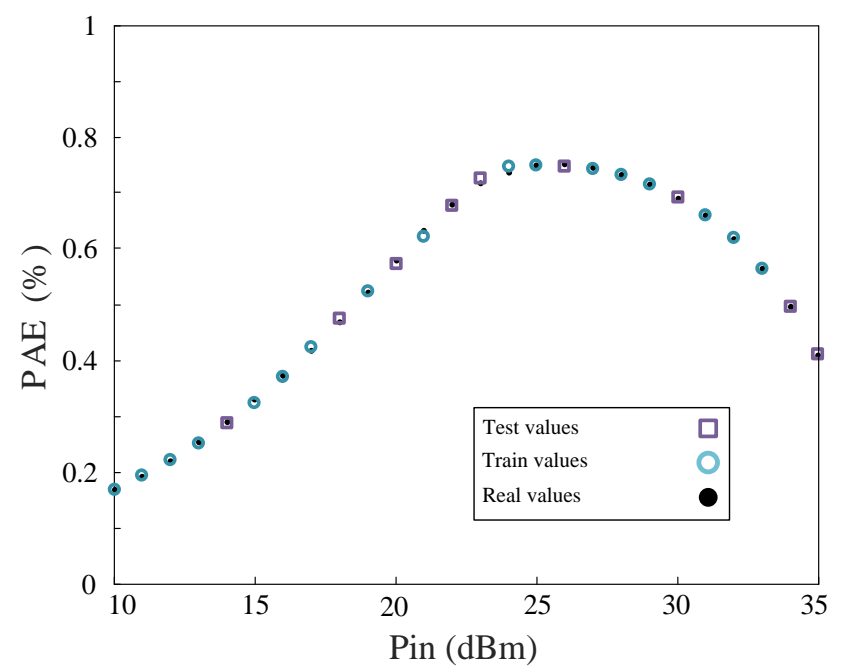

Fig. 7. Real and predicted PAE versus input power for training and testing data

\section{CONCLUSION}

An RBF network is proposed to model a linear class-F PA. Input power, DC power and output power of the PA are assumed as input and output variables of the RBF model. The RBF model results are compared with the MLP model. Normalization process is performed on the data set to enhance the accuracy of the presented model. The MAE and RMSE of 0.311 and 0.002 for the RBF network are obtained, respectively. Better than $84 \%$ and $92 \%$ improvements in $\mathrm{MAE} \%$ and RMSE have been achieved, so, the proposed RBF network is more accurate than the MLP model. According to the reliability of the presented model, it can be used to model the designed PA as a block or a sub circuit for circuit design. The same procedure can be performed on the other RF and microwave components to achieve their artificial neural network model. Modeling of the other microwave components and amplifiers using the presented procedure will be addressed in the future work.

TABLE III. TRAIN DATA OF THE Proposed RBF NETWork

\begin{tabular}{lllll}
\hline & $\begin{array}{l}\text { Input } \\
\text { Power } \\
{[\mathbf{d B m}]}\end{array}$ & $\begin{array}{c}\text { DC Power } \\
{[\mathbf{d B m}]}\end{array}$ & $\begin{array}{l}\text { Output } \\
\text { Power } \\
{[\mathbf{d B m}]}\end{array}$ & $\begin{array}{c}\text { Network } \\
\text { Output } \\
{[\mathbf{d B m}]}\end{array}$ \\
\hline 1 & 31.9866 & 37.3500 & 36.9400 & 36.9439 \\
2 & 26.9984 & 37.0100 & 36.2800 & 36.2652 \\
3 & 10.9691 & 33.4000 & 26.3800 & 26.4053 \\
4 & 46.9984 & 38.9500 & 40.7300 & 40.7333 \\
5 & 38.9982 & 37.7600 & 38.1600 & 38.1523 \\
6 & 32.9885 & 37.4100 & 37.0800 & 37.0805 \\
7 & 45.9988 & 38.7400 & 40.3900 & 40.3777 \\
8 & 44.9969 & 38.5800 & 40.0100 & 40.0356 \\
9 & 15.9988 & 34.5800 & 30.4600 & 30.4481 \\
10 & 10.0000 & 33.1600 & 25.5700 & 25.5395 \\
11 & 11.9866 & 33.6300 & 27.2000 & 27.2480 \\
12 & 20.9691 & 36.0500 & 34.2700 & 34.2050 \\
13 & 18.9982 & 35.4900 & 32.8500 & 32.8733 \\
14 & 30.9691 & 37.2800 & 36.8000 & 36.8003 \\
15 & 43.9967 & 38.4400 & 39.6300 & 39.6099 \\
16 & 37.9934 & 37.6900 & 37.9600 & 37.9284 \\
17 & 40.0000 & 37.8800 & 38.3900 & 38.4088 \\
18 & 35.9988 & 37.6200 & 37.5800 & 37.5798 \\
19 & 24.9969 & 36.8700 & 35.9800 & 35.9862 \\
20 & 27.9934 & 37.0800 & 36.4100 & 36.4024
\end{tabular}




\begin{tabular}{lllll}
21 & 14.9969 & 34.3000 & 29.6500 & 29.5788 \\
22 & 23.9967 & 36.7900 & 35.7700 & 35.8245 \\
23 & 42.9885 & 38.2900 & 39.2400 & 39.2297 \\
24 & 40.9691 & 38.0100 & 38.6400 & 38.6578 \\
25 & 28.9982 & 37.1500 & 36.5400 & 36.5393 \\
26 & 12.9885 & 33.8400 & 28.0200 & 28.0082 \\
27 & 41.9866 & 38.1700 & 38.9200 & 38.9260 \\
28 & 47.9996 & 39.1800 & 41.1100 & 41.1095 \\
29 & 16.9984 & 34.8900 & 31.2700 & 31.3330 \\
\hline \multicolumn{4}{c}{ REFERENCES }
\end{tabular}

[1] S.Y. Zheng, Z.W. Liu, Y.M. Pan, Y. Wu, W.S. Chan and Y. Liu, "Bandpass Filtering Doherty Power Amplifier With Enhanced Efficiency and Wideband Harmonic Suppression," IEEE Transactions on Circuits and Systems I: Regular Papers 63 (2016) 337-346. doi: 10.1109/TCSI.2016.2515419.

[2] R. Jos, "RF Monte Carlo calculation of power amplifier efficiency as function of signal bandwidth," International Journal of Microwave and $\begin{array}{lllll}\text { Wireless Technologies } & 8 & \text { (2016) } & 125-133 & \text { doi: }\end{array}$ 10.1017/S175907871500015X.

[3] Y. Ding, Y. X. Guo and F.L. Liu, "High-efficiency concurrent dual-band class-F and inverse class-F power amplifier," Electron. Lett. 47 (2011) 847-849, doi:10.1049/el.2011.1662.

[4] S. Gao, "High efficiency class-F RF/microwave power amplifiers," IEEE Microw. Mag. 7 (2006) 40-48, doi:10.1109/MMW.2006.1614233.

[5] K. Chen and D. Peroulis, "A 3.1-GHz class-F power amplifier with $82 \%$ power-added-efficiency," IEEE Microw. Wirel. Compon. Lett. 23 (2013) 436-438, doi:10.1109/LMWC.2013.2271295.

[6] N. Dawar, T. Sharma, R. Darraji, and F.M. Ghannouchi, "Linearisation of radio frequency power amplifiers exhibiting memory effects using direct learning-based adaptive digital predistoriton," IET Communications 10 (2016) 950-954, doi: 10.1049/iet-com.2015.1048.

[7] X. Zheng, L. Su, S. Hu, Z. Ye and J. He, "Legendre wavelet for power amplifier linearization," Analog Integrated Circuits and Signal Processing 84 (2015) 283-292, doi:10.1007/s10470-015-0544-9.

[8] G. Antonini, and A. Orlandi, "Gradient evaluation for neural networks based electromagnetic optimization procedures," IEEE Trans. Microw. Theory Techn. 48 (2000) 874-876, doi:10.1109/22.841892.

[9] S. Haykin, Neural Networks, 2nd edn. (Upper Saddle River, NJ Prentice- Hall, 1999).

[10] K. C. Lee and P. Gardner, "Neuro-fuzzy approach to adaptive digital predistortion," Electron. Lett. 40 (2004) 185-187, doi:10.1049/el:20040154.
[11] T. Liu, S. Boumaiza and M. Ghannouchi, "Dynamic behavioral modeling of $3 \mathrm{G}$ power amplifiers using real-valued time-delay neural networks," IEEE Trans. Microw. Theory Tech. 52 (2004) 1025-1033, doi:10.1109/TMTT.2004.823583.

[12] M. Hayati and A. Lotfi, "Modeling of compact microstrip resonator using neural network: Application to design of compact low pass filter with sharp cutoff frequency," Microw. Opt. Techn. Let. 53 (2011), doi:10.1002/mop.26008.

[13] G. H. Karimi, S. Babaei Sedaghat and R, Banitalebi, "Designing and modeling of ultra low voltage and ultra low power LNA using ANN and ANFIS for Bluetooth applications," Neurocomputing 120 (2013) 504508, doi:10.1016/j.neucom.2013.04.021.

[14] G. Kothapalli, "Artificial neural networks as aids in circuit design," Microelectr J. 26 (1995) 569-578, doi:10.1016/0026-2692(95)00019-E.

[15] M. Hayati, F. Shamma, S. Roshani and A. Abdipour, "Linearization design method in class-F power amplifier using artificial neural network," J. Comput. Electron. 13 (2014) 943-949, doi:10.1007/s10825014-0612-x.

[16] A. Mohammad, K. Anil and A. JA, "Design and Analysis of Microstrip Line Feed Toppled T Shaped Microstrip Patch Antenna using Radial Basis Function Neural Network," J. Electr. Eng. Technol. 10 (2015) 634-640, doi:10.5370/JEET.2015.10.2.634.

[17] M. Hui, T. Liu, M. Zhang, Y. Ye, D. Shen and X. Ying, "Augmented radial basis function neural network predistorter for linearisation of wideband power amplifiers," Electronics Letters 50 (2014) 877-879, doi: 10.1049/el.2014.0667.

[18] M. Aneesh, A. Kumar, A. Singh, K. Kamakshi, and J.A. Ansari, "Design and Analysis of Microstrip Line Feed Toppled T Shaped Microstrip Patch Antenna using Radial Basis Function Neural Network," Journal of Electrical Engineering and Technology 10 (2015) 634-640, doi: 10.5370/JEET.2015.10.2.634

[19] F. H. Raab, "An introduction to class-F power amplifiers," RF Design 19 (1996) 79-84.

[20] C. Zhao, J. Liu, F. Shen and Y. Yi, "Low power CMOS power amplifier design for RFID and the Internet of Things," Comput. Electr. Eng. (to be published 2015), doi:10.1016/j.compeleceng.2015.06.006.

[21] A. Panteli, M. Maragoudakis, and S. Gritzalis, "Privacy preserving data mining using radial basis functions on horizontally partitioned databases in the malicious model," Int. J. Artif. Intell. Tools 23 (2014) 1450007 , doi:10.1142/S021821301450007 